\title{
MISSION PERFORMANCE SIMULATION OF INTEGRATED HELICOPTER-ENGINE SYSTEMS USING AN AEROELASTIC ROTOR MODEL
}

\author{
Ioannis Goulos,, Panos Giannakakis, Vassilios Pachidis, Pericles Pilidis \\ Department of Power \& Propulsion \\ School of Engineering \\ Cranfield University \\ Bedfordshire, MK43 OAL, UK
}

\begin{abstract}
This paper presents an integrated approach, targeting the comprehensive assessment of combined helicopter-engine designs, within designated operations. The developed methodology comprises a series of individual modeling theories, each applicable to a different aspect of helicopter flight dynamics and performance. These relate to rotor blade modal analysis, threedimensional flight path definition, flight dynamics trim solution, aeroelasticity and engine performance. The individual mathematical models are elaborately integrated within a numerical procedure, solving for the total mission fuel consumption. The overall simulation framework is applied to the performance analysis of the Aérospatiale SA330 helicopter within two generic, twin-engine medium helicopter missions. An extensive comparison with flight test data on main rotor trim controls, power requirements and unsteady blade structural loads is presented. It is shown that, for the typical range of operating conditions encountered by modern twin-engine medium civil helicopters, the effect of operational altitude on fuel consumption is predominantly influenced by the corresponding effects induced on the engine, rather than on airframe-rotor performance. The implications associated with the implicit coupling between aircraft and engine performance, are discussed in the context of mission analysis. The potential to comprehensively evaluate integrated helicopterengine systems within complete three-dimensional operations, using modeling fidelity designated for main rotor design appli-
\end{abstract}

\footnotetext{
*Address all correspondence to this author: i.goulos@ cranfield.ac.uk
}

cations, is demonstrated. The proposed method essentially constitutes an enabler in terms of focusing the rotorcraft design process on designated operation types, rather than on specific sets of flight conditions.

$\begin{array}{ll}\text { NOMENCLATURE } \\ \begin{array}{ll}\text { Roman Symbols } \\ \Delta t\end{array} \\ \begin{array}{ll}\text { Mission analysis time-step, sec } \\ d_{\text {lat }}^{\text {min }}\left(x_{\text {lat }}^{\circ}\right)\end{array} \\ \begin{array}{l}\text { Cartesian length corresponding to a minute of lat- } \\ \text { itude for a latitudinal coordinate equal to } x_{\text {lat }}^{\circ}, m\end{array} \\ d_{\text {long }}^{\text {min }}\left(x_{\text {lat }}^{\circ}\right) & \begin{array}{l}\text { Cartesian length corresponding to a minute of lon- } \\ \text { gitude for a latitudinal coordinate equal to } x_{\text {lat }}^{\circ}, m\end{array} \\ F_{n} & \begin{array}{l}\text { Fuel burn calculated for the } n^{\text {th }} \text { mission iteration, } \\ k g\end{array} \\ h & \text { Rotor disc distance from ground, } m \\ M_{\text {intake }} & \text { Engine intake Mach number } \\ n & \text { Fuel burn iteration index } \\ P & \text { Per rotor revolution } \\ P_{\text {engine }} & \text { Engine shaft power, } k W \\ P_{\text {rotor }} & \text { Main rotor power required, } k W \\ R & \text { Rotor blade radius, } m \\ t & \text { Time-point within the mission, } \mathrm{sec} \\ V & \text { Flight speed, } m / \mathrm{sec} \\ w_{f} & \text { Engine fuel flow, } \mathrm{kg} / \mathrm{sec} \\ x_{\text {lat }}^{\circ} & \text { Latitudinal coordinate, } \mathrm{deg} \\ x_{\text {long }}^{\circ} & \text { Longitudinal coordinate, } \mathrm{deg}\end{array}$




\section{Greek Symbols}

$\begin{array}{ll}\varepsilon & \text { Mission fuel consumption tolerance } \\ \mu & \text { Advance ratio, } \frac{V}{\Omega R} \\ \Omega & \text { Main rotor nominal rotorspeed, } \mathrm{rad} / \mathrm{sec} \\ \theta_{0}, \theta_{1 c}, \theta_{1 s} & \text { Main rotor trim control angles, } \mathrm{deg} \\ \text { Acronyms } & \\ \text { ACARE } & \text { Advisory council for aeronautics research in Eu- } \\ & \text { rope } \\ \text { AGL } & \text { Above ground level } \\ \text { AUM } & \text { All up mass, } \mathrm{kg} \\ \text { EHOC } & \text { European helicopter operators' committee } \\ \text { FAR } & \text { Combustor fuel to air ratio } \\ \text { HPC } & \text { High-pressure compressor } \\ \text { IGE } & \text { In ground effect } \\ \text { ISA } & \text { International standard atmosphere } \\ \text { LPC } & \text { Low-pressure compressor } \\ \text { MTE } & \text { Mission task element } \\ \text { OAG } & \text { Oil \& gas } \\ \text { OEW } & \text { Operational empty weight, } \mathrm{kg} \\ \text { OGE } & \text { Out of ground effect } \\ \text { OPR } & \text { Overall pressure ratio } \\ \text { OW } & \text { Operational weight, } \mathrm{kg} \\ \text { SAR } & \text { Search \& rescue } \\ \text { SFC } & \text { Specific fuel consumption, } \mathrm{kg} / \mathrm{J} \\ \text { SOT } & \text { Stator outlet temperature, } \mathrm{K} \\ \text { WGS84 } & \text { World geodetic system dated in } 1984\end{array}$

\section{INTRODUCTION Background}

Flight dynamics simulation along with powerplant performance, mission analysis and design optimization, have always been important topics for the helicopter industry. These topics are now raising even more interest, as aspects related to fuel economy, chemical emissions and noise, gradually gain more importance in environmental and social impact assessments. Developments in technology within the $20^{t h}$ and the $21^{s t}$ century, mainly with respect to industrial as well as civil transport activities, have essentially led to a continuous worldwide rise in energy demand. Fossil fuel bound energy has been the dominant source explored and utilized for the satisfaction of this gradually increasing requirement. The progressively increasing rates of energy consumption, have inevitably resulted in the potential occurrence of fossil fuel depletion, as well as in the associated environmental impact due to the chemical emissions produced in the process [1].

The deployment of helicopters has been, until presently, focused mainly on activities such as civil transport, fire suppression, emergency medical evacuation, search \& rescue, oil \& gas and law enforcement. The aforementioned activities correspond to approximately $1,500,000$ flight hours annually, only with respect to the European airspace. This figure may seem insignificant when compared to the equivalent of 10,000,000 flight hours per year, as regards the European commercial airlines [2]. It is noted however that, a sharp growth of rotorcraft operations is expected in the forth-coming future.

The Advisory Council for Aeronautics Research in Europe (ACARE), in an attempt to manage the environmental impact of civil aviation, has set a number of goals which are to be achieved by 2020 . These goals include, among others, $50 \%$ reduction in $\mathrm{CO}_{2}$ emissions through drastic reduction of overall fuel consumption, relative to the 2000 level. According to Clarke [3], if reductions in the environmental impact of aircraft operations are to be realized, then either; (a) the number of operations must be reduced, (b) the type of aircraft must be changed, or (c) the aircraft operational rules and procedures must be changed.

As regards the helicopter industry, option (a) is not a viable path due to aforementioned expected growth in rotorcraft traffic. Considering option (b), Brooker [4] points out that, the timescales involved between the conception of an innovative aircraft design and the achievement of airworthiness certification can range from 20 to 50 years time. Thus, given the currently available time-frame, option (b) is not considered an effective response to the ACARE targets. It is therefore realized that, in terms of addressing the 2020 ACARE goals, emphasis needs to be placed towards the design and implementation of operational procedures leading to minimum required fuel burn and associated environmental impact [5].

It is noted however that, although the investigation of conceptual designs may not effectively lead to the realization of the relatively short-termed ACARE goals, it still is a viable path towards a longer term solution. Therefore, in order to effectively control the long-term environmental impact of civil rotorcraft aviation while simultaneously catering for the expected traffic growth, options concerning both conceptual design configurations as well as incorporated operational procedures, need to be thoroughly explored.

As regards the context of multidisciplinary rotorcraft design, both comprehensiveness as well as computational efficiency are deemed as prerequisites with respect to the employed design assessment approach. Key design variables, which might affect the overall performance of an integrated helicopter-engine system within a designated type of operation, have to be readily identifiable by the deployed formulation. An advanced level of simulation fidelity is therefore required, so that the behavior of the associated performance trade-off between designs optimized in a multidisciplinary manner, is accurately captured. The associated computational overhead has to be maintained within an acceptable threshold, so that the employed approach is applicable within an iterative optimization process. It is therefore realized that, an efficient compromise between modeling fidelity and computational efficiency has to be sought for. 


\section{Rotorcraft flight dynamics simulation models}

Padfield [6] has provided a three-level hierarchy of helicopter simulation models as a means of gauging the progress of attained simulation fidelity. Within level 1 modeling, a disclike representation of the main rotor is incorporated along with linear blade aerodynamics. The wake-induced velocity at the rotor is essentially expressed as a superposition of finite flow states within a $1^{\text {st }}$ order dynamic inflow formulation. A level 2 approach may employ individual-blade representation coupled with a more refined induced flow model. At level 3, the complete aeroelastic behavior of the main rotor is catered for. This necessitates the deployment of a detailed structural representation of the elastic blades (modal or finite element approach), coupled with unsteady, nonlinear blade element aerodynamics and three-dimensional rotor wake induced flow modeling (free or prescribed) [6].

Level 1-2 models are mainly deployed for the analysis of parametric trends as regards flying qualities and flight performance well within the limits of the operational envelope. Level 3 models are predominantly utilized for rotor design, unsteady rotor loads prediction and vibration analysis up to the limits defined by the safe flight envelope [6]. It is thus understood that, investigation of environmentally-friendly operational procedures within a well-defined operational envelope, can be catered for with the incorporation of a level 1-2 modeling approach. It is also emphasized however that, a multidisciplinary rotor design process essentially requires level 3 modeling fidelity, in order to identify the effects of key design parameters on the overall helicopter performance and unsteady rotor blade structural loads during flight. Models being currently employed for mission performance simulation $[2,5]$ belong to the $1^{\text {st }}$ level of Padfield's hierarchical paradigm. The implementation of higher order modeling fidelity in helicopter mission analysis applications, has until presently been hindered by the prohibitively large computational overhead associated with level 3 modeling.

D'Ippolito et al. [2] presented an integrated methodology for the simulation of a twin-engine light rotorcraft within a Category A take-off maneuver. Their mathematical approach included the use of the EUROPA rotorcraft code [7] and the engine performance simulation tool GSP [8]. Goulos et al. [5] subsequently extrapolated this methodology towards the simulation and multidisciplinary optimization of complete, three-dimensional rotorcraft operations for fuel burn, chemical emissions and ground noise impact. The EUROPA code essentially utilizes steady-state nonlinear blade element aerodynamics within a disc-like representation of the rotor disc based on rigid body dynamics. Thus, the simulation approach deployed in Refs. [2,5] essentially belongs to the $1^{\text {st }}$ level of Padfield's three-fold hierarchy of simulation models. This constitutes the overall approach, inapplicable towards the task of rotor design which among others may require reasonably accurate estimates of rotor blade structural loads during flight.

\section{Scope of present work}

In appreciation of the requirement for a cost-effective methodology capable of addressing point (c) raised by Clarke [3] with respect to the rotorcraft section of civil aviation, a comprehensive helicopter mission analysis approach utilizing an unsteady aeroelastic rotor model has been developed. The proposed method is capable of assessing the helicopter's main rotor behavior at the $3^{\text {rd }}$ level of Padfield's hierarchical paradigm of simulation models. The approach is therefore applicable to the task of rotor design and can be utilized for the investigation of conceptual configurations. The overall methodology is based upon a numerical procedure combining various individual mathematical formulations, each addressing a specific aspect of helicopter flight dynamics and performance. The individual mathematical theories apply to the tasks of rotor blade structural analysis, treatment of main rotor blade flexibility, nonlinear flight dynamics helicopter trim, three-dimensional flight path definition and engine performance simulation.

The integrated methodology is applied towards the analysis of two generic helicopter operations: a Search \& Rescue (SAR) and an Oil \& Gas (OAG) mission. The deployed model is based on the popular Aérospatiale SA330 twin-engine medium helicopter. Extensive comparisons with flight test data on main rotor trim controls, power requirements and unsteady blade structural loads have been waged. The time-variations of various engine performance parameters during flight are presented and analyzed.

The simulations carried out suggest that for the typical range of operating conditions encountered by modern twin-engine medium civil helicopters, the effect of operational altitude on fuel consumption is predominantly influenced by the corresponding effects induced on the engine rather than on airframe-rotor performance. It is shown that, thorough identification of the most power-demanding conditions within a complete helicopter operation, cannot be properly addressed without catering for the implicit coupling between engine and main rotor performance through the aircraft's time-dependent All Up Mass (AUM). The potential to deploy a level 3 simulation modeling approach for the performance evaluation of integrated helicopter-engine designs within complete, three-dimensional missions is demonstrated. The developed methodology essentially acts as enabler towards focusing the design process on the overall performance within designated operation types, rather than on specific sets of flight conditions with a somewhat arbitrarily defined aircraft AUM.

\section{NUMERICAL FORMULATION}

The integrated modeling approach employed for the simulation of complete rotorcraft operations, comprises a finite series of consecutive analyses, each applicable to a different aspect of flight dynamics and performance. The developed simulation 
framework comprises the Lagrangian rotor blade modal analysis presented in Ref. [9], a flight path profile analysis based on the World Geodetic System dated in 1984 (WGS 84), the nonlinear trim procedure solving for the aeroelastic behavior of the main rotor blades presented in Ref. [10] and an engine off-design performance analysis [11]. The individual modeling methodologies are combined within an elaborate integral procedure, solving for the unknown initial aircraft AUM. It is noted that the initial AUM essentially comprises the helicopter's Operational Empty Weight (OEW), the unknown required mission on-board fuel supplies $(F)$, and any necessary payload-equipment. The helicopter's OEW plus the necessary payload-equipment weight, constitute the Operational Weight (OW)

\section{WGS 84 flight path model}

The task of simulating effectively "real world" rotorcraft mission profiles requires three-dimensional treatment of the rotorcraft operational trajectory. For that reason, a flight path definition tool has been developed which allows for specification of a wide variety of "real world" rotorcraft operations. A complete mission is structured in terms of pre-defined mission task elements. Each mission task element represents a standardized procedure which is tailored according to the designated operation specifications. Examples of mission task elements include idle, hover, vertical climb/descent, straight and level flight (cruise), climbing turns, take-off/landing maneuvers etc.

The nature of the described implementation requires an effective approach for the translation of coordinates defined in a global geographical system (latitude-longitude) to the Cartesian level. Higher order polynomial expressions have been structured, describing the mathematical correspondence of a single minute of latitude/longitude to Cartesian length in meters. The offered expressions have been derived based on the global representation employed by WGS 84 [12], it being the standard coordinate frame of reference considering earth geography definition. The derived polynomials are provided in $6^{\text {th }}$ decimal accuracy below:

$$
\begin{gathered}
d_{\text {lat }}^{\min }\left(x_{\text {lat }}^{\circ}\right)=1.597401 E-11\left(x_{\text {lat }}^{\circ}\right)^{6}- \\
5.368544 E-07\left(x_{\text {lat }}^{\circ}\right)^{4}-6.766806 E-13\left(x_{\text {lat }}^{\circ}\right)^{3}+ \\
5.606089 E-03\left(x_{\text {lat }}^{\circ}\right)^{2}+3.330305 E-09 x_{\text {lat }}^{\circ}+1.842911 E+03
\end{gathered}
$$

$$
\begin{gathered}
d_{\text {long }}^{\min }\left(x_{\text {lat }}^{\circ}\right)=-4.682085 E-11\left(x_{\text {lat }}^{\circ}\right)^{6}+ \\
6.766856 E-06\left(x_{\text {lat }}^{\circ}\right)^{4}- \\
2.955679 E-13\left(x_{\text {lat }}^{\circ}\right)^{3}- \\
2.807881 E-01\left(x_{\text {lat }}^{\circ}\right)^{2}+1.439889 E-09 x_{\text {lat }}^{\circ}+1.855346 E+03
\end{gathered}
$$

where $x_{\text {lat }}^{\circ}$ is the latitudinal coordinate in degrees and $d_{\text {lat }}^{\text {min }}, d_{\text {long }}^{\text {min }}$ is the Cartesian length in meters, corresponding to a minute of latitude and longitude respectively. It can be noticed from Eqs. $(1,2)$ that $d_{\text {lat }}^{\text {min }}$ and $d_{\text {long }}^{\text {min }}$ are only functions of $x_{\text {lat }}^{\circ}$. Equations $(1,2)$ are utilized within this paper for the translation of coordinates from the global to the Cartesian system and viceversa.

\section{Rotor blade modal analysis}

A minimum potential energy approach based on Lagrangian kinematics is utilized in order to obtain the natural vibration characteristics of the main rotor blades for flap-lag-torsion [9]. The rotor blade is treated as a continuous rotating system of nonuniform structural properties. A finite series of assumed deformation functions, based on Bernoulli-Euler beam and classical torsion theory, is used for the approximation of the time variations of the blade's strain and kinetic energy for each degree of freedom. Integral expressions describing the generalized centrifugal forces and moments exerted on the blade are incorporated. Inserting the expressions for strain, kinetic energy and generalized centrifugal forces/moments into Lagrange's equation of motion, results in the formation of the eigenproblem which is solved with customary matrix techniques, thus leading to the required natural frequencies and mode shapes for each degree of freedom.

\section{Aeroelastic rotor model}

The incorporated mathematical rotor model employs the numerical approach presented in Ref. [10] for the treatment of rotor blade flexibility in the time domain. The method caters for the inclusion of all nonlinear inertial terms associated with large blade deflections as well as the helicopter's three-dimensional motion. The rotor model is coupled with the Peters - He finite state induced flow model [13] and the Leishman - Beddoes unsteady nonlinear blade element aerodynamics model [14]. Lookup tables describing the steady-state aerodynamic aerofoil response, are deployed for the calibration of the Leishman - Beddoes model.

Careful treatment of the induced flow terms arising from shed wake circulation has been carried out, so that they are not duplicated by both induced flow and unsteady aerodynamics models. The dynamic response of the elastic blades to the imposed aerodynamic and inertial external loads is estimated in the time domain using a $5^{\text {th }}$ order accurate numerical evaluation scheme of the convolution integral. Rotor-fuselage aerodynamic interaction effects are accounted for through deployment of a steady-state potential flow method applied to the fuselage geometry. Ground effect treatment is carried out by applying the closed form expressions derived by Cheeseman and Bennet [15]. 


\section{Nonlinear trim procedure}

The aircraft fuselage is treated dynamically as a rigid body with six degrees of freedom (three translations $\&$ three rotations) using the inertial tensors and the OW provided in Ref. [6]. Experimentally derived look-up tables extracted from Ref. [6], are employed for the estimation of fuselage force and moment coefficients as functions of incidence and sideslip angles. Lookup tables are also utilized for the prediction of the aerodynamic behavior of the horizontal and vertical stabilizers. Steady-state aerofoil characteristics along with a $1^{s t}$ order dynamic inflow formulation [16] are used for the prediction of the tail-rotor performance.

A globally convergent Newton-Raphson approach is incorporated in order to obtain the required trim control and fuselage attitude angles for any set of flight conditions. The numerical scheme is applied towards the solution a linearized version of Euler's equations of motion for rigid body kinematics. The trim linearization assumption dictates that, the time-derivatives of the flight state vector elements are essentially zero. The main and tail rotors are marched simultaneously in time using the initial condition of nonexistent circulatory wake. The time-marching process continues until a once-per-rev periodic condition with respect to the main rotor mean induced flow is achieved. Having obtained flow periodicity, a finite series of main rotor rotations is carried out where the main and tail rotor hub-wind forces and moments are averaged in time in order to acquire mean representative values to be used for the trim procedure.

\section{Engine performance simulation}

The engine performance model (TURBOMATCH) used for the present work has been developed and refined at Cranfield University over a number of decades [11]. Turbomatch is based on zero-dimensional aero-thermodynamic analysis employing discrete component maps. The employed method essentially solves for the mass and energy balance between the various engine components. Turbomatch has been previously deployed in several studies available in the literature for the prediction of design and off-design performance of gas turbine engines [17, 18]. For the scope of the present analysis, the engine is assumed to be operating exclusively at steady-state off-design conditions.

\section{Mission breakdown}

Figure 1 presents a flow-chart which demonstrates the numerical procedure employed for the simulation of complete rotorcraft operations. The overall process involves the break-down of each designated mission task element, into discrete segments with a pre-defined time-step $\Delta t$. The helicopter is assumed to be operating in trim and the engine in steady-state off-design during each segment of duration $\Delta t$. This assumption is valid, considering that the primary focus of the structured numerical approach is the estimation of the total mission fuel consumption.

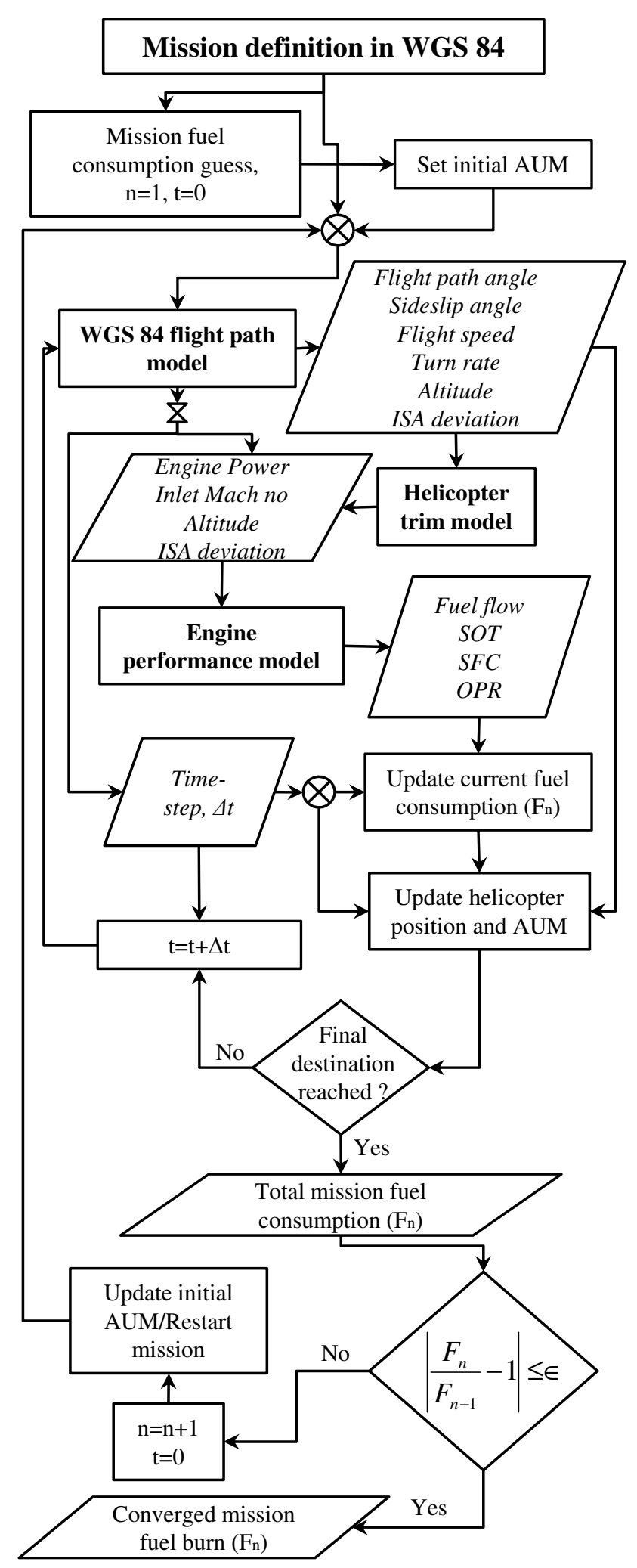

FIGURE 1. Integrated mission analysis numerical procedure flowchart 
Figure 1 shows that the mission analysis process is initiated $(n=1)$ with a user-defined guess as regards the overall mission fuel consumption. The initial rotorcraft AUM is subsequently determined based on that initial guess of the on-board fuel supplies. Depending on the mission definition and the corresponding segment time-point $t$, the flight path model determines the helicopter flight conditions. The nonlinear rotorcraft trim model subsequently trims the helicopter, thus determining among others, the engine shaft power requirements. The engine performance model subsequently establishes the engine operating point based on the shaft power requirements and the corresponding inlet conditions. Therefore, the engine fuel flow is determined among other off-design performance parameters. The time-dependent fuel consumption corresponding to time-point $t$, is calculated by applying a numerical time integration scheme over the mission segments corresponding to $0 \leq \tau \leq t$. This value is subtracted from the initial AUM at time $t$ in order to simulate the helicopter's gradual weight reduction during the course of the mission. The helicopter's spatial location is also updated based on the flight conditions dictated by the flight path model.

Having completed a first-pass $(n=1)$ along the entire mission, the estimated value of total fuel consumption is compared with the initial fuel burn guess. If the obtained error is within a user-defined tolerance $\varepsilon$, the process is halted. In the case that the acquired error exceeds $\varepsilon$, the initial AUM is updated based on the calculated total mission fuel consumption $F_{n}$. The overall process is re-iterated in a fixed-point manner until the error in the calculated fuel consumption is below $\varepsilon$ as shown in Fig. 1.

\section{RESULTS \& DISCUSSION}

The aircraft selected for the case study presented in this paper, has been modeled after the Aérospatiale SA330 Puma. The SA330 is a twin-engine medium helicopter equipped with two Turbomeca Turmo IV-C turboshaft engines rated at $1163 \mathrm{~kW}$ maximum contingency power. Table 1 presents the rotorcraft model design parameters. The Turmo IV-C engine is equipped with a single-spool gas generator including a single-stage axial followed by a centrifugal compressor. The engine configuration is outlined in Table 2 . The maximum contingency power setting has been selected as the design point for the respective Turbomatch model. The model has been matched at design point conditions with public domain data [19] in terms of Specific Fuel Consumption $(S F C)$ with an accuracy of $0.3 \%$.

The configuration of the SA330 as well as its performance characteristics have been extensively documented and analyzed in Refs. [6, 20], thus further elaboration shall be omitted. A detailed description of the Turbomeca Turmo engine family can be found in Ref. [19].
TABLE 1. Aérospatiale SA330 rotorcraft model design parameters

\begin{tabular}{lcc}
\hline Design parameter & Value & Unit \\
\hline OEW & 3536 & $\mathrm{~kg}$ \\
OW & 5805 & $\mathrm{~kg}$ \\
Number of blades & 4 & - \\
Blade radius & 7.53 & $\mathrm{~m}$ \\
Blade chord & 0.54 & $\mathrm{~m}$ \\
Blade twist & 0.14 & $\mathrm{rad}$ \\
Rotorspeed & 27 & $\mathrm{rad} / \mathrm{sec}$ \\
\hline
\end{tabular}

TABLE 2. Turbomeca Turmo IV-C engine model parameters

\begin{tabular}{lcc}
\hline Design parameter & Value & Unit \\
\hline Maximum shaft power $\left(P_{\text {engine }}\right)$ & 1163 & $\mathrm{~kW}$ \\
LPC pressure ratio (Axial) & 1.2 & - \\
HPC pressure ratio (Centrifugal) & 4.83 & - \\
Air mass flow $(W)$ & 5.9 & $\mathrm{~kg} / \mathrm{sec}$ \\
Stator outlet temperature $(S O T)$ & 1308 & $\mathrm{~K}$ \\
Combustor fuel to air ratio $(F A R)$ & 0.022 & - \\
Specific fuel consumption $(S F C)$ & $107.06 \cdot 10^{-9}$ & $\mathrm{~kg} / \mathrm{J}$ \\
\hline
\end{tabular}

\section{Rotor blade modal analysis}

Figure 2 presents the calculated rotor resonance chart for the full-scale, articulated rotor of the SA330 helicopter using the Lagrangian approach presented in Ref. [9]. The specific articulated rotor design has been extensively described in Ref. [20], thus further discussion on the rotor configuration will not be carried out. Simulation results from CAMRAD [21] with regards to flap-lag-torsion blade frequencies at nominal rotorspeed are also included. The natural frequency predictions made with CAMRAD have been extracted from Ref. [20]. The solid and broken lines correspond to the Lagrangian method predictions (Lagr.) while the markers signify CAMRAD calculations.

Good agreement between the Lagrangian method and CAMRAD simulations can be observed with respect to the predicted flap $(1 \mathrm{~F}-3 \mathrm{~F})$, lag $(1 \mathrm{~L}-2 \mathrm{~L})$ and torsion $(1 \mathrm{~T})$ resonant frequencies at nominal rotorspeed $(\Omega=27 \mathrm{rad} / \mathrm{sec})$. The acquired natural frequencies along with corresponding mode shapes, are utilized within the remainder of this paper for the approximation of the blade's dynamic response to any aerodynamic or inertial excitation. 

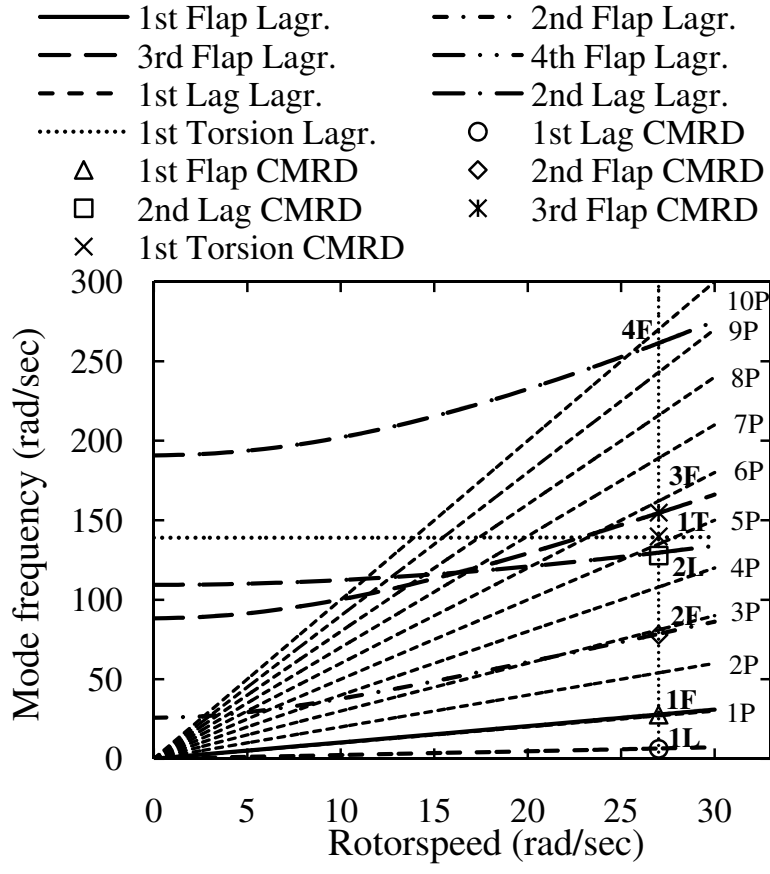

FIGURE 2. Resonance chart for the SA330 helicopter main rotor blades - Comparison with CAMRAD results from Ref. [20]

\section{Helicopter flight dynamics trim analysis}

Figures 3, 4 present trim controls and power requirement predictions for the main rotor based on simulations performed for the SA330 helicopter for straight and level flight. The numerical formulations described in sections "Aeroelastic rotor model" and "Nonlinear trim procedure" have been utilized in order to obtain trim solutions based on the unsteady, aeroelastic behavior of the articulated rotor. Results are presented as functions of advance ratio $\mu=\frac{V}{\Omega R}$ from hover $(\mu=0)$ to high-speed flight $(\mu \approx 0.4)$. Comparisons with flight test data extracted from Ref. [20] have also been included for validation purposes.

Figures 3, (a) and (b) present trim values of main rotor power requirement $P_{\text {rotor }}$ and collective pitch angle $\theta_{0}$ respectively. Good correlation between the integrated model predictions and flight test data can be observed regarding both trim outputs. Agreement is best for $\mu \geq 0.15$ where the flow-field in the vicinity of the main rotor begins to be dominated by the freestream component. Small discrepancies between predictions and experimental data are noted with respect to $\mu \leq 0.15$ considering both trim outputs. This indicates a potential overestimation of the induced losses at low-speed flight conditions.

Figures 4, (a) and (b) present trim values of lateral $\left(\theta_{1 s}\right)$ and longitudinal $\left(\theta_{1 c}\right)$ cyclic pitch angles respectively. Fair agreement is observed between predictions and flight test data considering both cyclic control inputs within the entire range of advance ratios. The simulations suggest that rotor wake impinge-
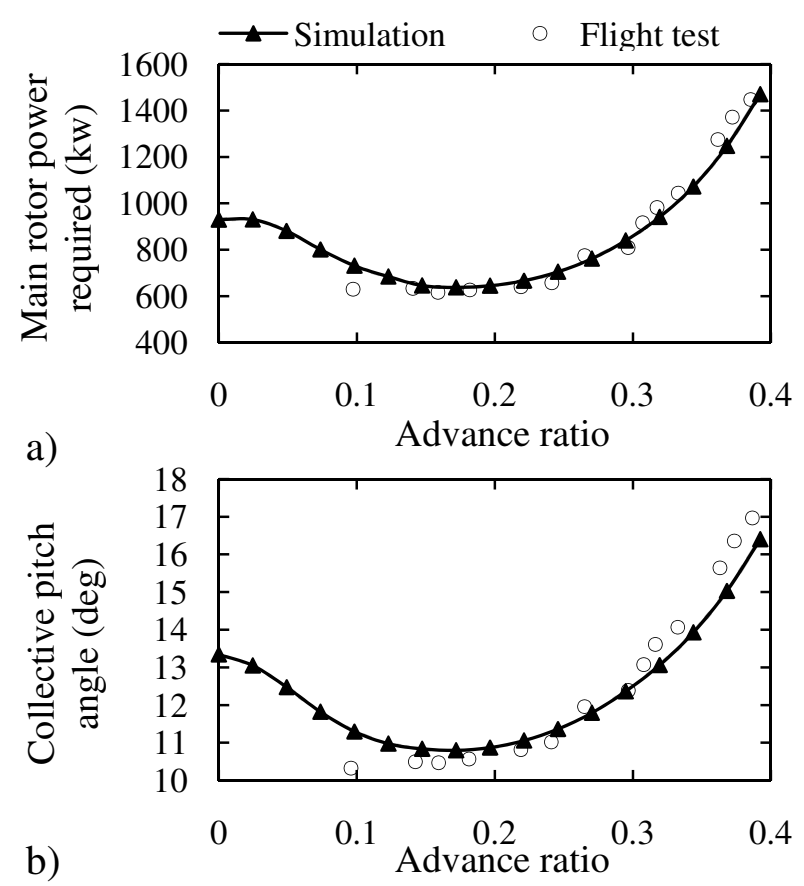

FIGURE 3. Flight dynamics trim results for the SA330 helicopter comparison with flight test data extracted from Ref. [20]: (a) Main rotor power required $P_{\text {rotor }},\left(\right.$ b) Collective pitch angle $\theta_{0}$
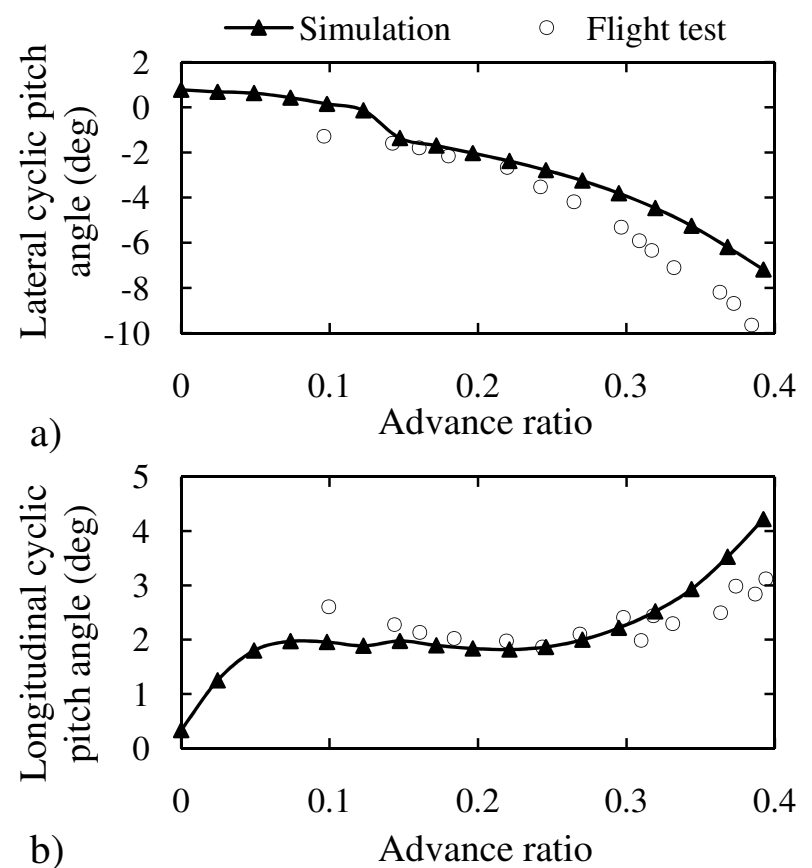

FIGURE 4. Flight dynamics trim results for the SA330 helicopter comparison with flight test data extracted from Ref. [20]: (a) Lateral cyclic pitch angle $\theta_{1 s}$, (b) Longitudinal cyclic pitch angle $\theta_{1 c}$ 
ment on the horizontal stabilizer occurs for $\mu \geq 0.15$ leading to large positive fuselage pitching moment. The control system thus compensates with a corresponding decrease in the lateral cyclic pitch control angle $\theta_{1 s}$ as shown in Fig. 4, (a). The flight test data suggest that rotor wake impingement interaction begins for lower advance ratios $(\mu \geq 0.1)$, since no corresponding decrease is observed in $\theta_{1 s}$ for $\mu \geq 0.1$.

\section{Unsteady rotor blade structural loads prediction}

Figures $5-8$ present correlations between predictions made with the present aeroelastic rotor model and flight test measurements of unsteady flapwise/chordwise bending and torsional moments for the full-scale articulated rotor of the SA330 helicopter. Results are presented for $\mu=0.307$ and $\mu=0.321$. The simulations correspond to trim solutions obtained for the respective values of advance ratio using the trim model described in section "Nonlinear trim procedure" of this paper. Comparisons of flapwise bending moment are presented for two blade radial locations corresponding to $r / R=0.35$ and $r / R=0.55$ respectively. Chordwise bending moment correlations are presented for $r / R=0.73$ while torsional moment correlations correspond to $r / R=0.33$. The superimposed flight test data have been extracted from from Ref. [20].

Figures 5-6 present comparisons between simulations and measurements of unsteady blade flapwise bending moment for $\mu=0.307$ and $\mu=0.321$ respectively. Results are presented for $r / R=0.35$ (Figs. 5, 6, (a)) and $r / R=0.55$ (Figs. 5, 6, (b)). Good correlation can be observed for both values of advance ratio between predictions and flight tests with respect to both radial locations and both values of advance ratio. It can be noticed that the behavior of $3 \mathrm{P}$ and $4 \mathrm{P}$ oscillatory loading is accurately predicted, both in terms of magnitude and phase considering both radial locations. Agreement is best for $\mu=0.321$.

Figures 7, (a) and (b) present correlations between simulations and measurements of unsteady blade chordwise bending moment for $\mu=0.307$ and $\mu=0.321$ respectively. Results are presented for $r / R=0.73$. The agreement can be considered fair in the sense that the overall magnitude of the oscillatory waveform has been captured. The higher frequency components of the waveform do not appear to have been accurately predicted. The larger discrepancies observed in comparison to the flap bending case, are attributed to insufficiently accurate aerofoil data with respect to drag coefficients.

Figures 8, (a) and (b) present correlations between simulations and measurements of unsteady blade torsional moment for $\mu=0.307$ and $\mu=0.321$ respectively. Comparisons are presented for $r / R=0.33$. Good agreement can generally be observed for both values of advance ratio for which results are presented. It is noted that, the amplitude as well as lower-frequency harmonic content of oscillatory torsional loading has been fairly accurately captured. Agreement is best for $\mu=0.321$.

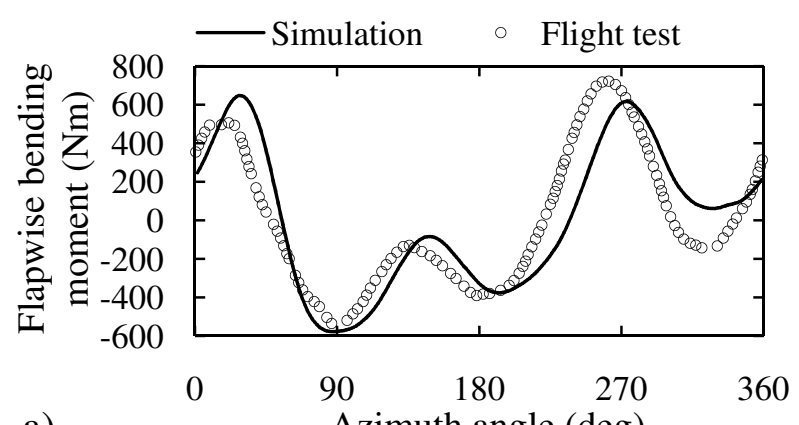

a) Azimuth angle (deg)

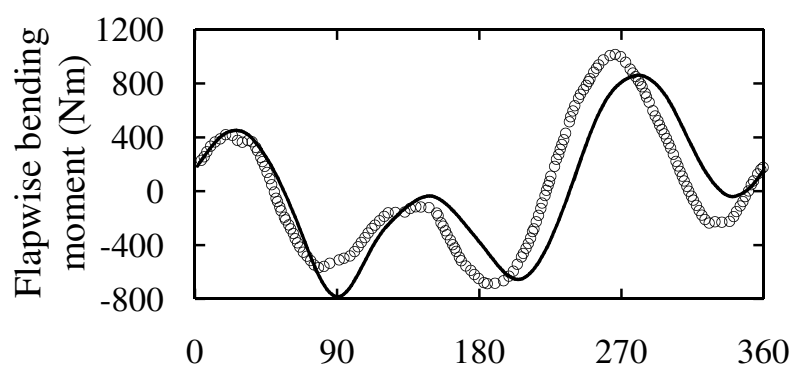

b) Azimuth angle (deg)

FIGURE 5. Unsteady flapwise blade bending moment for the SA330 helicopter rotor, $\mu=0.307$ - comparison with flight test data extracted from Ref. [20]: (a) $r / R=0.35$, (b) $r / R=0.55$
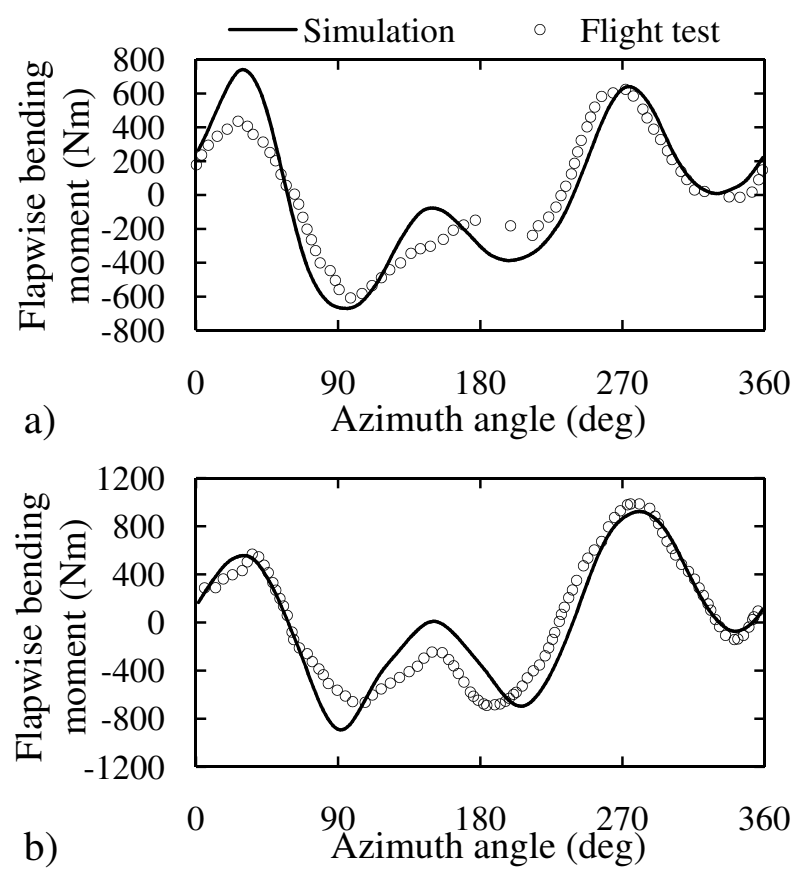

FIGURE 6. Unsteady flapwise blade bending moment for the SA330 helicopter rotor, $\mu=0.321$ - comparison with flight test data extracted from Ref. [20]: (a) $r / R=0.35$, (b) $r / R=0.55$ 


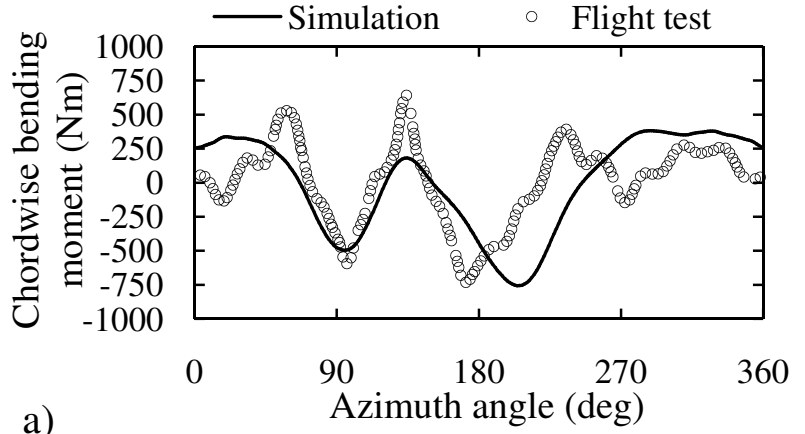

a)

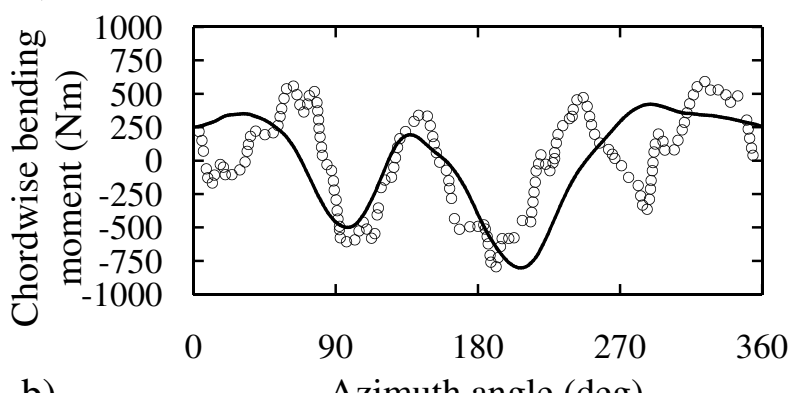

b)

Azimuth angle (deg)

FIGURE 7. Unsteady chordwise blade bending moment for the SA330 helicopter rotor, $r / R=0.73$ - comparison with flight test data extracted from Ref. [20]: (a) $\mu=0.307$, (b) $\mu=0.321$
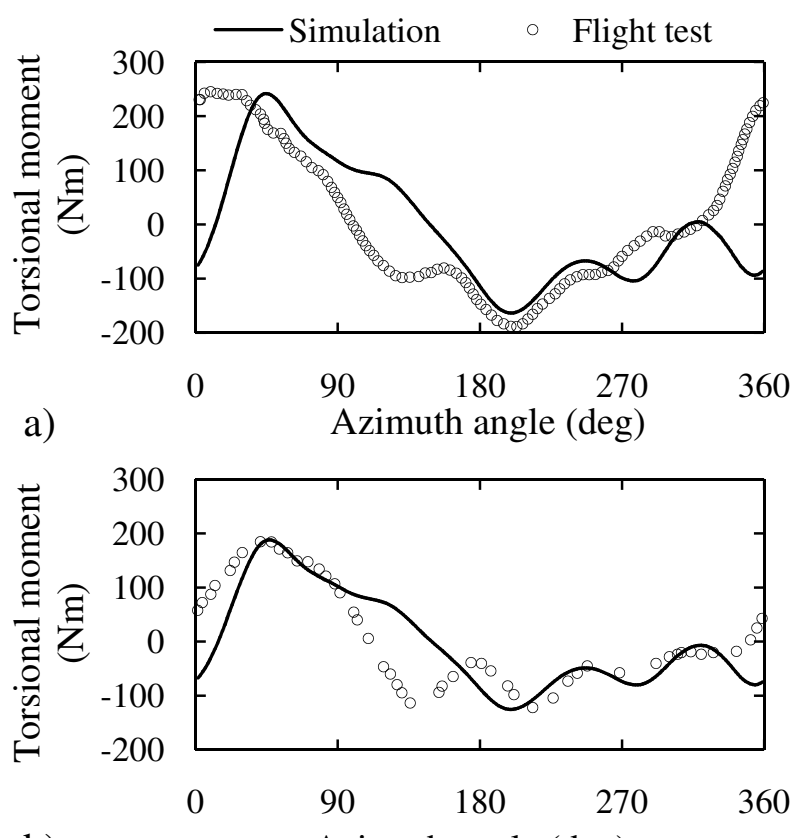

b)

Azimuth angle (deg)

FIGURE 8. Unsteady torsional blade moment for the SA330 helicopter rotor, $r / R=0.33$ - comparison with flight test data extracted from Ref. [20]: (a) $\mu=0.307$, (b) $\mu=0.321$

\section{Engine performance trim analysis}

Figures $9-10$ present the respective engine performance parameters as functions of advance ratio. The operating conditions correspond to the flight dynamics trim analysis presented in the respective section of this paper. Results are presented for atmospheric conditions of zero ISA deviation, at an absolute altitude of $100 \mathrm{~m}$. The intake mach number $M_{\text {intake }}$ is essentially defined by the designated advance ratio $\mu$.

It is shown that, all of the presented engine performance parameters, essentially follow the classic "bucket-shape" curve which is dictated by the power requirement of the main rotor as shown in Fig. 3, (a). It is noted that a small ram compression effect on engine performance is present in the results presented in Figs. 9 - 10. Figure 9, (a) shows that, the required engine shaft power $P_{\text {engine }}$ is roughly $500 \mathrm{~kW}$ for $\mu=0$ as well as for for $\mu \approx 0.33$. The latter value of $\mu$ corresponds to $M_{\text {intake }} \approx 0.2$ which essentially leads to an increase of inlet total pressure of the order of $2.7 \%$ due to ram compression. The predicted change in maximum engine pressure is around $0.5 \%$ upwards. The overall pressure ratio produced solely by the compressors however, is reduced by approximately $2.1 \%$ leading to a corresponding decrease in compressor work. It is noted that ram temperature rise may increase the compressor work for a given pressure ratio. With regards to the designated operating conditions and engine configuration however, the former effect is predominant leading to a reduction to the power absorbed from the gas generator turbine for compression. The corresponding increase in air mass flow $W$ due to ram compression is of the order of $0.94 \%$. The aforementioned effects essentially lead to a reduction in engine fuel flow of the order of $1.5 \%$ for $\mu \approx 0.33$ in comparison to the hovering case $(\mu=0)$ while maintaining identical shaft power output $P_{\text {engine }}$.

\section{Helicopter mission analysis}

Two generic, three-dimensional missions, representative of modern twin-engine medium helicopter operations have been defined: a search \& rescue (SAR) and an oil \& gas (OAG) mission. The incorporated operational procedures in terms of geographical location selection, deployed airspeed, altitude, climb/descent rates and idle times have been defined in collaboration with the European Helicopter Operator's Committee (EHOC). The geographical representation in terms of global coordinates, along with the deployed operational procedures, are illustrated in Figs. 11, 12 for the SAR and OAG case respectively.

The SAR mission schedule assumes that the helicopter takes-off from Stockholm Arlanda airport in Sweden and subsequently travels towards a designated location above the Baltic Sea. The helicopter is then assumed to engage in a search pattern in order to trace and pick up citizens in distress from a location corresponding to an assumed naval incident. After successfully locating the citizens in distress, the helicopter SAR crew picks 


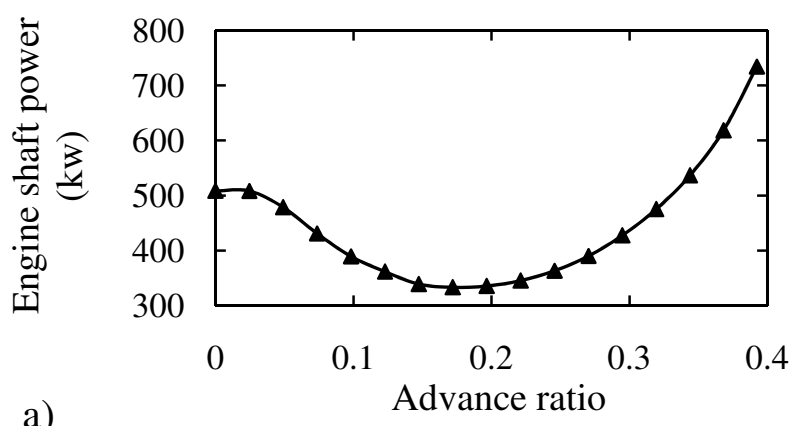

a)

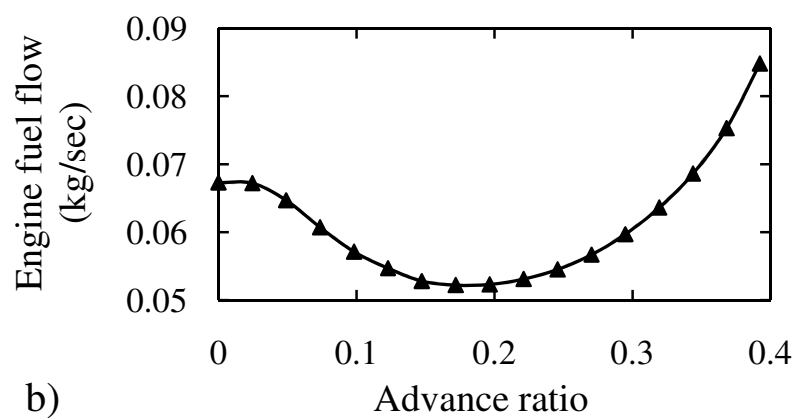

FIGURE 9. Engine performance trim results for the SA330 helicopter: (a) Shaft power $P_{\text {engine }}$, (b) Fuel flow $w_{f}$
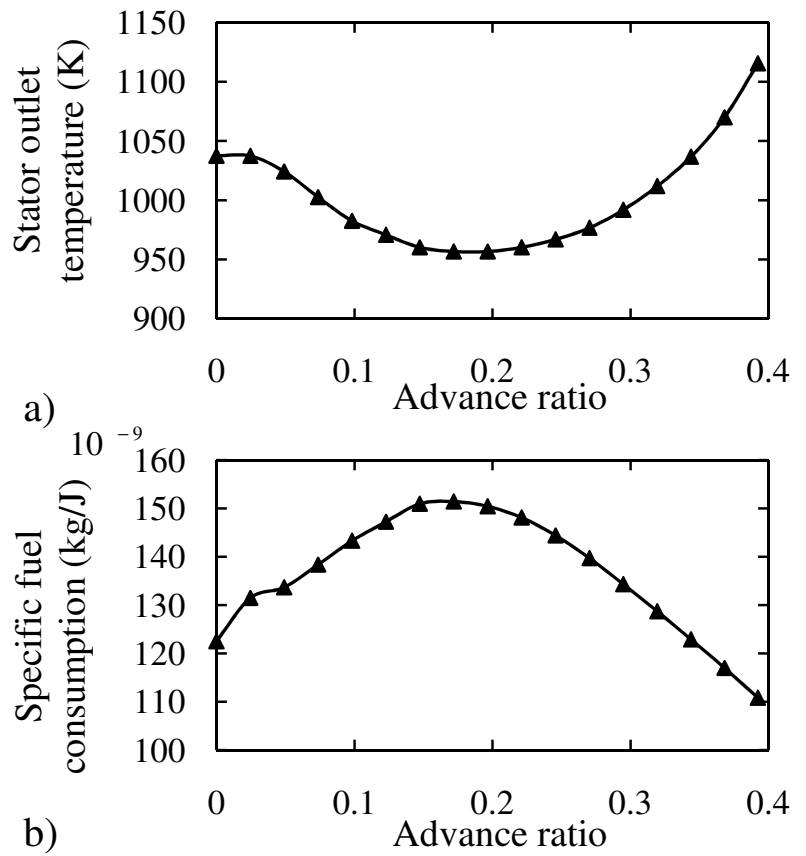

FIGURE 10. Engine performance trim results for the SA330 helicopter: (a) Stator outlet temperature (SOT), (b) Specific fuel consumption $(S F C)$ them up while hovering over the imaginary incident zone. The patients are then transported to Högbergsgatan hospital where the helicopter lands on the respective helipad where it drops-off the patients and subsequently returns to Stockholm Arlanda airport.

The OAG mission schedule assumes that the helicopter takes-off from De Kooy airfield in Den Helder, Netherlands, while carrying a specified payload. The helicopter subsequently travels towards a designated offshore oil/gas platform (oil rig 1) located in the North Sea where it lands and drops-off the onboard payload. The helicopter then travels towards a second offshore oil/gas platform (oil rig 2) where it picks-up another useful payload and subsequently returns to the original De Kooy airfield in Den Helder.

Climb and descent rates are held fixed at $5 \mathrm{~m} / \mathrm{sec}$ and 3.5 $\mathrm{m} / \mathrm{sec}$ respectively. A time-step $\Delta t$ of $5 \mathrm{sec}$ is used for the each individual mission segment. All coordinated turns are executed with a turn rate of $5^{\circ} / \mathrm{sec}$, with the exception of fine tuning the helicopter's orientation where the turn rate is defined based on the orientation error and the mission time-step $\Delta t$. During idle operation, the overall helicopter power requirements are assumed to be equal to $20 \%$ of maximum contingency engine shaft power.

Figures 11-12, (b) present the time variations of the helicopter's AGL altitude and flight speed for the SAR and OAG mission respectively. As regards the SAR mission, Fig. 11, (b) shows that the helicopter spends almost half of the mission time engaged in the SAR pattern where the deployed flight speed and AGL altitude is $30 \mathrm{~m} / \mathrm{sec}$ and $60 \mathrm{~m}$ respectively. With respect to the OAG operation, it can be observed that the overall mission comprises 3 cruise task elements. The second cuise element is a relatively short one, since the two oil rigs are located within a distance of only $8.6 \mathrm{~km}$.

The performance of the integrated helicopter-engine system is assessed throughout the course of both missions using the integrated methodology described in section "Mission breakdown" of this paper, applied to the Aérospatiale SA330 configuration (Tables 1, 2). The total parameters in terms of range, time and fuel consumption are outlined in Table 3 for both missions. It is noted that, a comparison of the calculated mission range using the flight path model described in section "WGS 84 flight path model" with calculations performed using the virtual globe/geographical information program "Google earth", revealed a relative error of the order of $0.05 \%$ and $0.1 \%$ for the SAR and OAG missions respectively. This indicates the very good predictive qualities of the polynomial expressions provided by Eqs. $(1,2)$.

Figures $13-14$ and $15-16$ demonstrate the variation of various engine performance parameters as functions of mission time for the SAR and OAG missions respectively. The idle segments can be distinguished as conditions associated with low values of engine shaft power, fuel flow, and SOT but relatively high SFC. Climbing forward flight along with hover, are identified as the most demanding trim settings in terms of engine shaft power. 

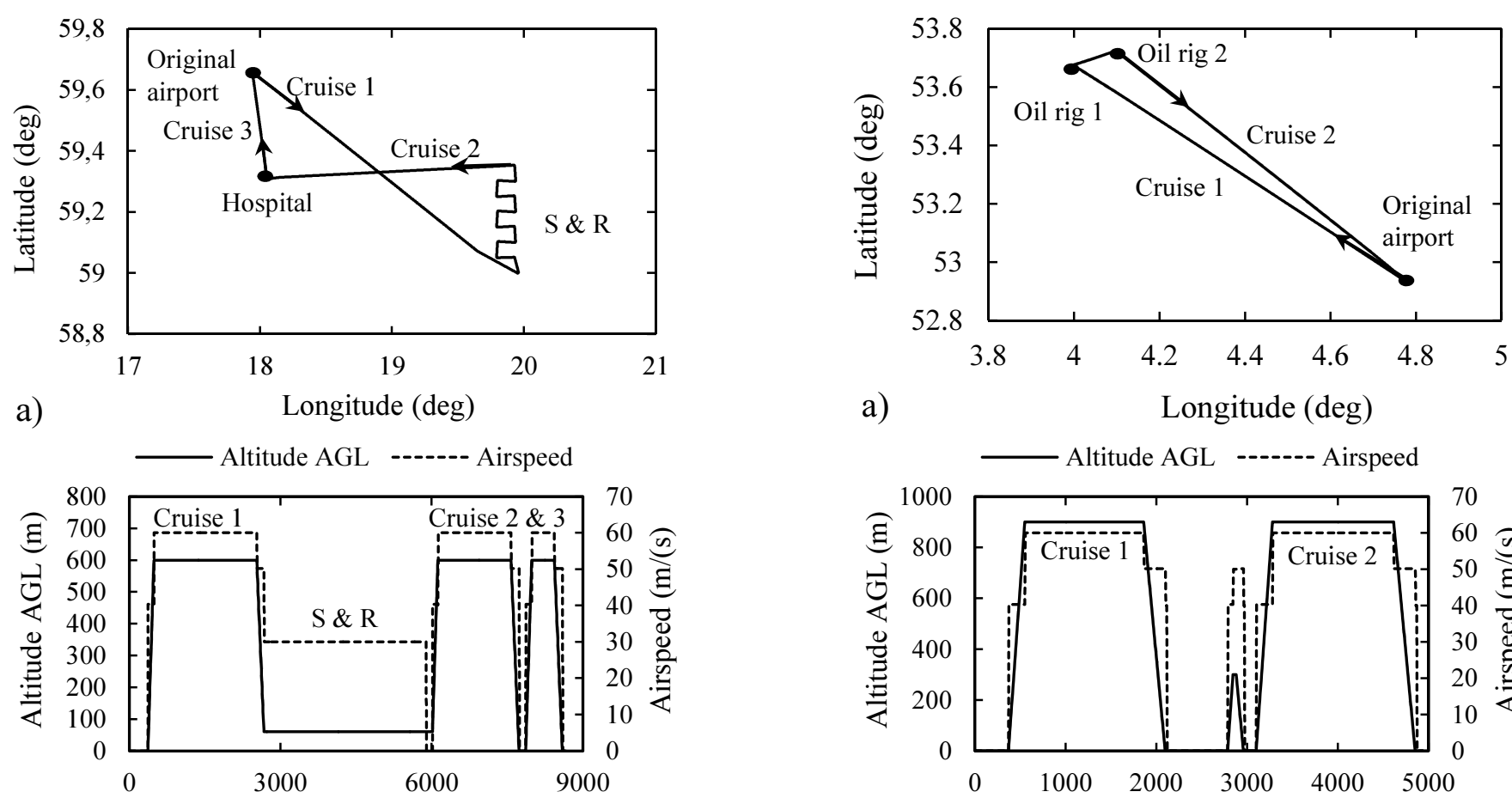

b) Mission time (sec)

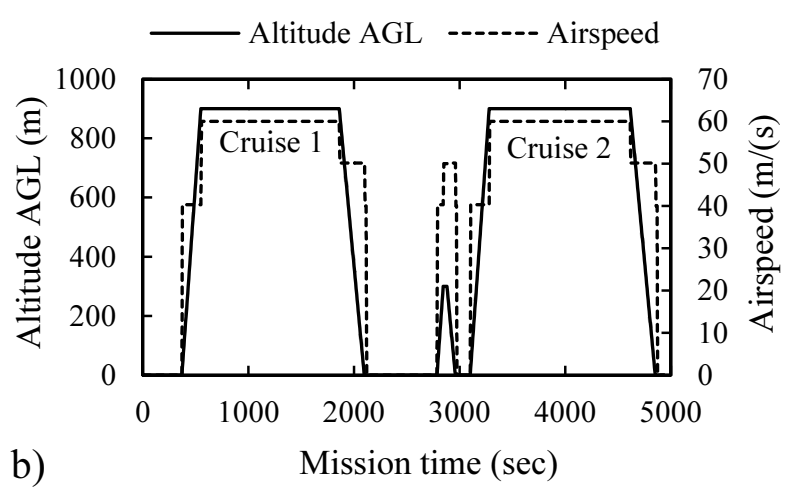

FIGURE 11. Search \& Rescue (SAR) mission: (a) Geographical definition, (b) Time-variations of deployed operational airspeed and AGL altitude

TABLE 3. Total mission parameters.

\begin{tabular}{lccc}
\hline Mission parameter & SAR & OAG & Unit \\
\hline Range & 370.1 & 206.8 & $\mathrm{~km}$ \\
Time & 8660 & 4935 & $\mathrm{sec}$ \\
Total fuel consumption & 1022.2 & 551.1 & $\mathrm{~kg}$ \\
\hline
\end{tabular}

For identical atmospheric conditions, the relationship between hover and climbing forward flight, in terms of rotor power requirement, depends on the aircraft AUM. A large AUM, due to either on-board fuel supplies or useful payload, essentially requires higher main rotor thrust settings. This results in increased rotor induced flow, hence induced losses. With respect to hovering conditions, the main rotor power requirement is essentially dominated by the induced losses. At higher values of advance ratio however, the rotor induced flow is significantly reduced and thus the additional induced losses associated with a larger aircraft AUM have a smaller impact on aircraft performance. Therefore, a large AUM may impose a more significant penalty on the rotor power requirement in hover, rather than climbing or forward flight, due to the associated reduction in mean induced flow. 

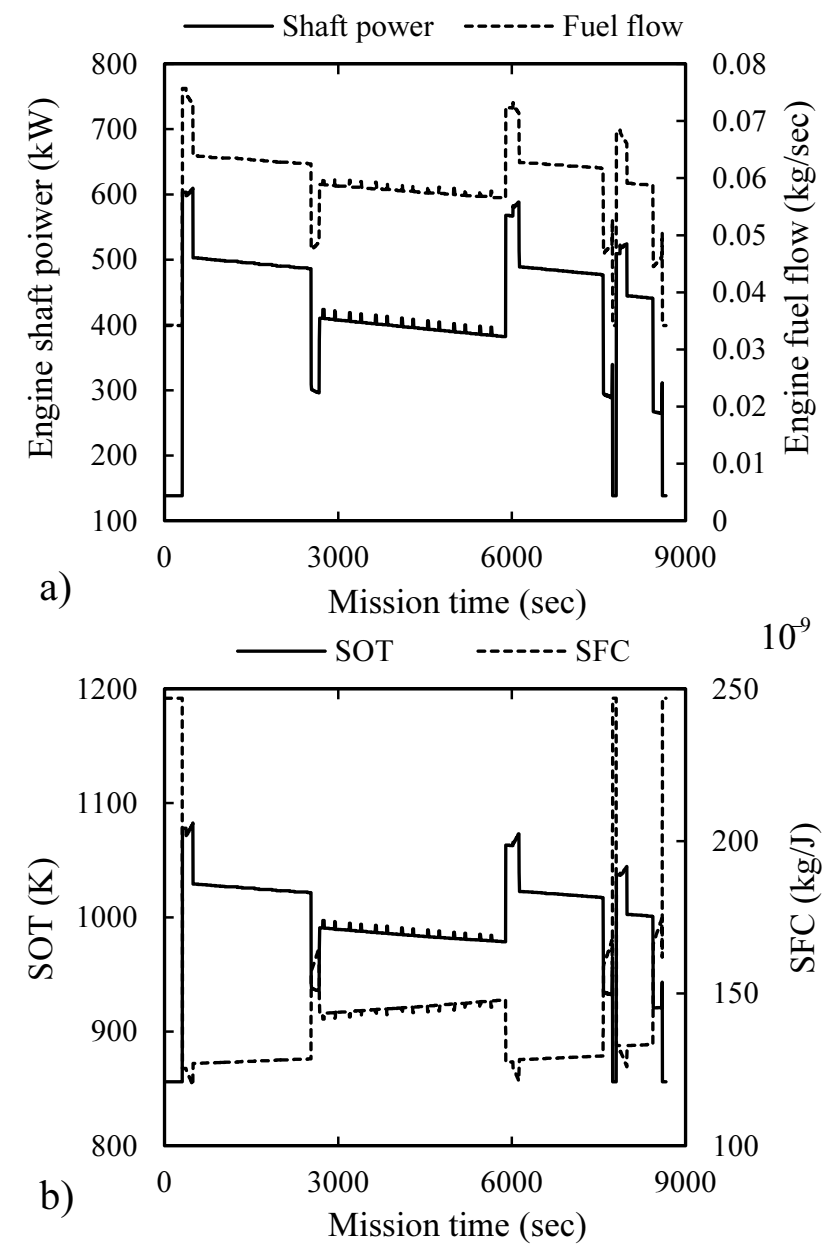

FIGURE 13. Engine performance parameters for the SAR mission: (a) Shaft power $P_{\text {engine }}-$ Fuel flow $w_{f}$, (b) Stator outlet temperature $S O T$ - Specific fuel consumption $S F C$

however, Figs. 13, 15, (a) show that hovering flight is still among the flight conditions with the highest power requirement considering both investigated operations.

An interesting behavior is observed with respect to climbing/descending forward flight, as regards both designated operations. Figures 13, 15, (a) show that during climb, there is a gradual increase in engine shaft power. This is due to the gradual decrease in air density, leading to increased induced flow through the main rotor disc for a given thrust setting, resulting in a corresponding rise in induced losses. This is not the case however with respect to fuel flow. Fuel flow is actually shown to decrease with altitude, despite the rise in engine shaft power. This is attributed to the gradually decreasing ambient temperature which gives rise to an increased referred rotational speed. This leads to increased values of $O P R$ and SOT (Figs. 13, 15, (b)) which combine to increase the engine thermal efficiency and thus decrease the overall $S F C$. Thus, the favorable altitude effect on en-
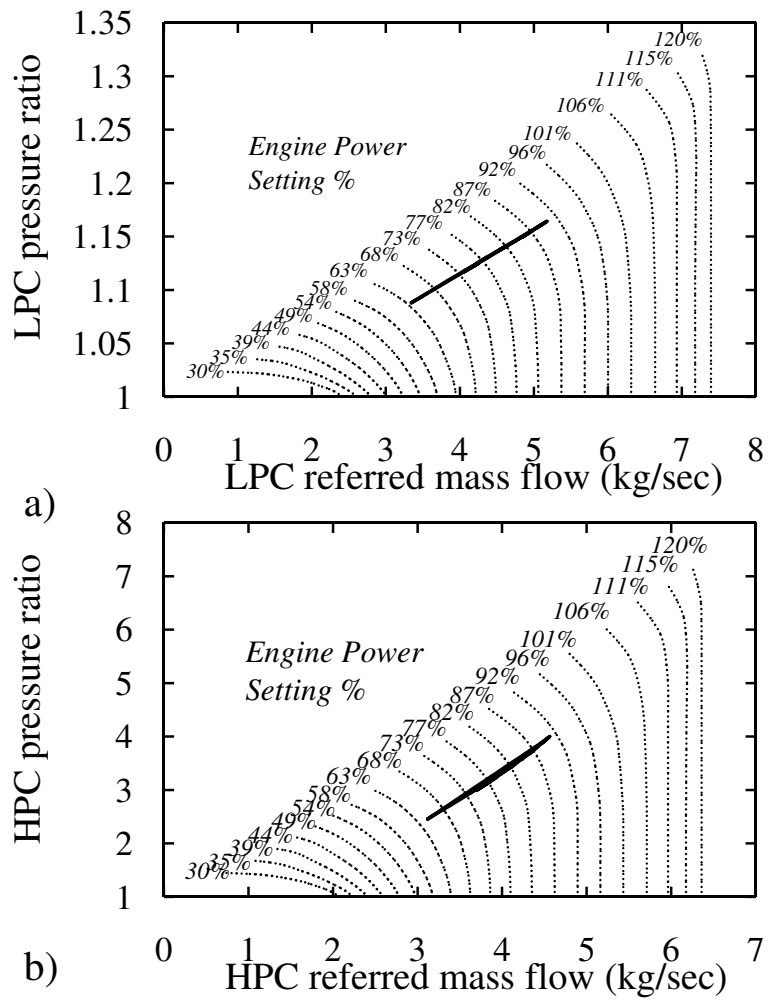

FIGURE 14. Engine performance parameters for the SAR mission: (a) LPC running line, (b) HPC running line

gine thermal efficiency, dominates over the penalizing influence on airframe-rotor performance, leading to an overall reduction in required fuel flow. The reverse behavior is observed for descending forward flight.

It is interesting to note the magnitude of the helicopter's gradual weight reduction effect due to the associated fuel consumption, on the engine shaft power requirement as well as on the various engine performance parameters presented for both missions. This indicates once again the importance of accurately estimating the initial aircraft AUM. This aspect is especially significant for long-range missions where the large weight of the on-board fuel supplies can influence the overall mission fuel consumption considerably. It is understood that, overestimation of the on-board fuel supplies may lead to a penalty in fuel consumption, while underestimation may render the designated mission unfeasible. It can thus concluded that, accurate prediction of the overall mission fuel consumption ahead of flight, is a key parameter in addressing the aspect of fuel economy.

Figures 14, 16 present the Low-Pressure Compressor (LPC) and High-Pressure Compressor (HPC) operating points during the course of SAR and OAG mission respectively. It is observed that both LPC and HPC operating points are located within a well-defined region of representative running lines for both operations. This is due to the fact that the engine essentially encoun- 

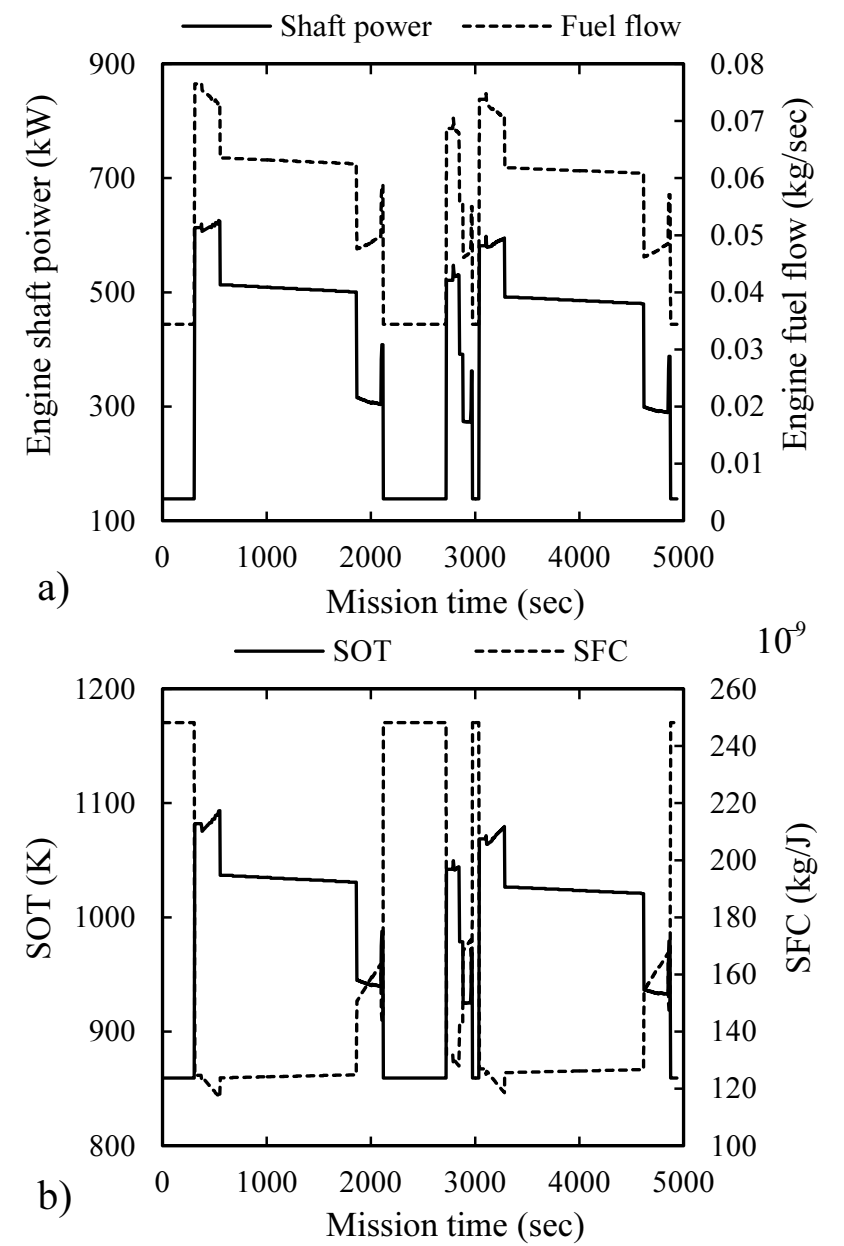

FIGURE 15. Engine performance parameters for the SAR mission: (a) Shaft power $P_{\text {engine }}-$ Fuel flow $w_{f}$, (b) Stator outlet temperature $S O T$ - Specific fuel consumption $S F C$

ters a relatively low range of mach numbers $\left(0 \leq M_{\text {intake }} \leq 0.2\right)$ within the operational envelope defined by a typical twin-engine medium helicopter mission. Therefore, the location of the operating points within the corresponding compressor map is predominantly affected by the engine shaft power. Thus, for zero ISA deviation, the LPC and HPC operating points within a generic helicopter mission are found to be closely spaced around the region defined by the typical engine running line for sea-level static conditions.

\section{CONCLUSIONS}

A comprehensive helicopter mission analysis approach, utilizing an unsteady aeroelastic rotor model, has been presented. The proposed method has been shown to be capable of performing flight dynamics assessments at the $3^{\text {rd }}$ level of Padfield's hierarchy of simulation models. The approach is therefore applicable
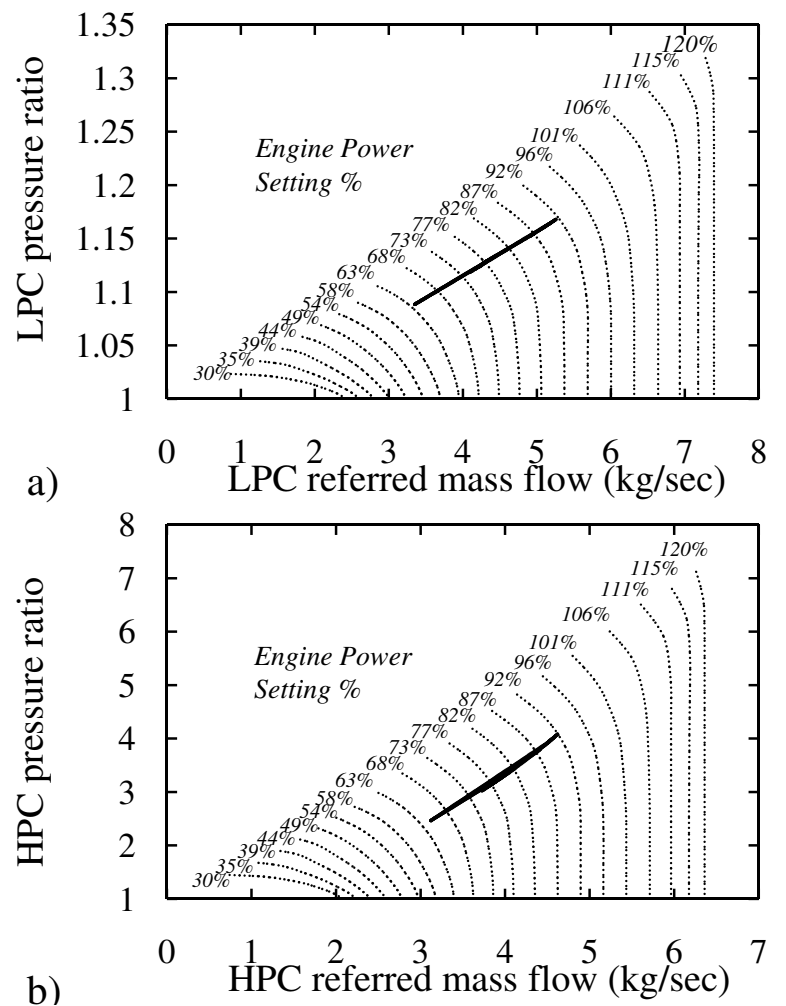

FIGURE 16. Engine performance parameters for the SAR mission: (a) LPC running line, (b) HPC running line

to the task of rotor design, and therefore to the investigation of conceptual configurations as well as innovative operational procedures. The integrated approach has been successfully applied towards the performance analysis of a twin-engine medium helicopter, modeled after the Aérospatiale SA330. Good agreement has been shown between predictions and flight test measurements with regards to main rotor trim controls, power requirements and unsteady blade structural loads. Two generic helicopter missions, representative of modern twin-engine medium rotorcraft operations have been analyzed.

The simulations carried out suggest that, for the range of operating conditions encountered by modern twin-engine medium rotorcraft, the favorable altitude effect on engine thermal efficiency, dominates over the respective penalizing influence on airframe-rotor performance. It has been argued that, thorough identification of the most power-demanding conditions within a complete helicopter operation, cannot be properly addressed without catering for the implicit coupling between engine and airframe-rotor performance through the aircraft's timedependent AUM. It has been demonstrated that correct estimation of the overall mission fuel consumption ahead of flight, is a key parameter in addressing the aspect of mission fuel economy. The LPC and HPC operating points within a generic twin-engine medium helicopter mission, have been found to be closely spaced 
around the region defined by the typical engine running line for sea-level static conditions. The potential to deploy a level 3 simulation modeling approach for the evaluation of helicopter-engine integrated systems within complete, three-dimensional missions has been demonstrated. The developed methodology can essentially be regarded as enabler in terms of placing the focus of the design process of integrated helicopter-engine systems, to the overall performance within designated operation types, rather than on specific sets of flight conditions with a somewhat arbitrarily defined aircraft AUM.

\section{ACKNOWLEDGMENTS}

The authors would like to acknowledge Dr Vishal Sethi from the department of Power \& Propulsion of Cranfield University for his insightful advice and continuing support.

\section{REFERENCES}

[1] Goulos, I., Mohseni, M., Pachidis, V., d'Ippolito, R., and Stevens, J., "Simulation framework development for helicopter mission analysis," Proceedings of ASME Turbo Expo 2010, Vol. 3, Glasgow, Scotland, 14-18 June 2010, pp. 843852.

[2] d'Ippolito, R., Stevens, J., Pachidis, V., Berta, A., Goulos, I., and Rizzi, C., "A Multidisciplinary Simulation Framework for Optimization of Rotorcraft Operations and Environmental Impact," EngOpt 2010, 2nd International Conference on Engineering Optimization, Lisbon, Portugal, 6-9 September 2010.

[3] Clarke, J.-P., "The role of advanced air traffic management in reducing the impact of aircraft noise and enabling aviation growth," Journal of Air Transport Management, Vol. 9(3), 2003, pp. 161-165.

[4] Brooker, P., "Civil Aircraft Design Priorities: Air Quality? Climate Change? Noise?" The Aeronautical Journal, Vol. 110(1110), 2005, pp. 517-532.

[5] Goulos, I., Pachidis, V., D'Ippolito, R., Stevens, J., and Smith, C., "An Integrated Approach for the Multidisciplinary Design of Optimum Rotorcraft Operations," Journal of Engineering for Gas Turbines and Power, Vol. 134(9), 2012.

[6] Padfield, G. D., Helicopter Flight Dynamics, Blackwell Publishing, 2nd ed., 2007.

[7] Serr, C., Hamm, J., Toulmay, E., Polz, G., Langer, H. J., Simoni, M., andA. Russo, M. B., Vozella, A., Young, C., Stevens, J., Desopper, A., and Papillier, D., "Improved Methodology for Take-Off and Landing Operational Procedures - The RESPECT Programme," 25th European Rotorcraft Forum, Rome, Italy, 14-16 September 1999.

[8] Visser, W. and Broomhead, M., "GSP: A generic objectoriented gas turbine simulation environment," Tech. Rep.
NLR-TP-2000-267, National Aerospace Laboratory NLR, 2000.

[9] Goulos, I., Pachidis, V., and Pilidis, P., "Lagrangian Formulation for the Rapid Estimation of Helicopter Rotor Blade Vibration Characteristics," Article submitted to Journal of Aircraft, 2012.

[10] Goulos, I., Pachidis, V., and Pilidis, P., "Helicopter Rotor Blade Flexibility Simulation for Aeroelasticity and Flight Dynamics Applications," Article submitted to Journal of the American Helicopter Society, 2012.

[11] Macmillan, W. L., Development of a Module Type Computer Program for the Calculation of Gas Turbine Off Design Performance, Ph.D. thesis, Department of Power and Propulsion, Cranfield University, 1974.

[12] European Organization for the Safety of Air Navigation (EUROCONTROL) and Institute of Geodesy and Navigation (IfEN), Brussels, Belgium, WGS 84 IMPLEMENTATION MANUAL, 1998.

[13] Peters, D. A., Boyd, D. D., and He, C. J., "FiniteState Induced-Flow Model for Rotors in Hover and Forward Flight," Journal of the American Helicopter Society, Vol. 34(4), 1989, pp. 5-17.

[14] Leishman, J. G. and Beddoes, T., “A Semi-Empirical Model for Dynamic Stall," Journal of the American Helicopter Society, Vol. 34(3), 1989, pp. 3-17.

[15] Cheeseman, I. C. and Bennet, W. E., "The effect of the Ground on a Helicopter Rotor in Forward Flight," Tech. Rep. R \& M No 3021, Aeronautical Research Council, 1957.

[16] Peters, D. A. and HaQuang, N., "Dynamic Inflow for Practical Applications," Journal of the American Helicopter Society, Vol. 33(4), 1988, pp. 64-68.

[17] Li, Y. G., Marinai, L., Gatto, E. L., Pachidis, V., and Pilidis, P., "Multiple-Point Adaptive Performance Simulation Tuned to Aeroengine Test-Bed Data," Journal of Propulsion and Power, Vol. 25(3), 2009, pp. 635-641.

[18] Pachidis, V., Pilidis, P., Marinai, L., and Templalexis, I., "Towards a full two dimensional gas turbine performance simulator," Aeronautical Journal, Vol. 111(1121), 2007, pp. 433-442.

[19] Gunston, B., Jane's Aero-engines, Jane's Information Group, 1996.

[20] Bousman, W. G., Young, C., Toulmay, F., Gilbert, N. E., Strawn, R. C., Miller, J. V., Costes, T. H. M. M., and Beaumier, P., "A Comparison of Lifting-Line and CFD Methods with Flight Test Data from a Research Puma Helicopter," Tech. Rep. TM-110421, National Aeronautics and Space Administration, October 1996.

[21] Johnson, W., "A comprehensive Analytical Model of Rotorcraft Aerodynamics and Dynamics Vol. I: Theory Manual," Tech. rep., Johnson Aeronautics, 1988. 
2013-06-30

\title{
Mission performance simulation of pÿintegrated helicopter engine systems using an aeroelastic rotor model
}

\author{
Goulos, loannis
}

ASME

Ioannis Goulos, Panos Giannakakis, Vassilios Pachidis and Pericles Pilidis, Mission

pÿperformance simulation of integrated helicopter engine systems using an aeroelastic rotor model. ASME Turbo Expo 2013: Turbine Technical Conference and Exposition, 3-7 June 2013, San Antonio, Texas, USA. Paper No. GT2013-94798

https://dspace.lib.cranfield.ac.uk/handle/1826/12242

Downloaded from Cranfield Library Services E-Repository 